\title{
Erratum to: UDP-glucuronosyltransferase 1A1 is the principal enzyme responsible for puerarin metabolism in human liver microsomes
}

\author{
Cheng-Feng Luo - Bin Cai • Ning Hou • Mu Yuan • Shi-Ming Liu • \\ Hong Ji $\cdot$ Long-Gen Xiong $\cdot$ Wei Xiong $\cdot$ Jian-Dong Luo $\cdot$ Min-Sheng Chen
}

(C) Springer-Verlag 2012

Erratum to: Arch Toxicol

DOI 10.1007/s00204-012-0874-7

In the original publication of this article, unfortunately there is a mistake in Table 1: column 4: position 3 should be $1^{\prime \prime}$ and not $12^{\prime \prime}$.
The online version of the original article can be found under doi: 10.1007/s00204-012-0874-7.

C.-F. Luo · B. Cai · S.-M. Liu · L.-G. Xiong · M.-S. Chen $(\bowtie)$

The Second Affiliated Hospital of Guangzhou Medical

University, Guangzhou Institute of Cardiovascular Disease,

250, Changgangdong Road, Guangzhou 510260, China

e-mail: gzminsheng@vip.163.com

N. Hou · M. Yuan · H. Ji · W. Xiong · J.-D. Luo

Guangzhou Medical University, 195, Dongfengxi Road,

Guangzhou 510182, China
Table $1{ }^{1} \mathrm{H}$ and ${ }^{13} \mathrm{C}$ NMR spectral data for M2 (400 MHz for ${ }^{1} \mathrm{H}$, $100 \mathrm{MHz}$ for ${ }^{13} \mathrm{C}$ )

\begin{tabular}{|c|c|c|c|c|c|}
\hline Position & $\begin{array}{l}\delta_{\mathrm{H}} \text { mult } \\
(J \text { in } \mathrm{Hz})\end{array}$ & $\begin{array}{l}\delta_{\mathrm{C}} \\
\text { mult }\end{array}$ & Position & $\begin{array}{l}\delta_{\mathrm{H}} \text { mult } \\
(J \text { in } \mathrm{Hz})\end{array}$ & $\begin{array}{l}\delta_{\mathrm{C}} \\
\text { mult }\end{array}$ \\
\hline 2 & $8.42 \mathrm{~s}$ & 153.7 & $\begin{array}{l}\text { 7-O- } \\
\text { GlcUA }\end{array}$ & & \\
\hline 3 & & 123.7 & $1^{\prime \prime}$ & $5.02 \mathrm{~d}(6.8)$ & 101.4 \\
\hline 4 & & 175.4 & $2^{\prime \prime}$ & & 73.5 \\
\hline 5 & $\begin{array}{r}8.07 \mathrm{~d} \\
(8.8)\end{array}$ & 127.1 & $3^{\prime \prime}$ & & 75.9 \\
\hline 6 & $\begin{array}{l}7.24 \mathrm{~d} \\
(9.3)\end{array}$ & 118.8 & $4^{\prime \prime}$ & & 72.1 \\
\hline 7 & & 159.9 & $5^{\prime \prime}$ & & 74.0 \\
\hline 8 & & 114.0 & $6^{\prime \prime}$ & & 172.4 \\
\hline 9 & & 157.7 & 8-C-Glc & & \\
\hline 10 & & 116 & $1^{\prime \prime}$ & $\begin{array}{l}4.88 \mathrm{~d} \\
\quad(10.0)\end{array}$ & 74.0 \\
\hline $1^{\prime}$ & & 122.6 & $2^{\prime \prime}$ & & 69.8 \\
\hline $2^{\prime}$ & $\begin{array}{l}7.41 \mathrm{~d} \\
(8.4)\end{array}$ & 129.9 & $3^{\prime \prime}$ & & 79.0 \\
\hline $3^{\prime}$ & $\begin{array}{l}6.81 \mathrm{~d} \\
(8.4)\end{array}$ & 115.4 & $4^{\prime \prime}$ & & 67.6 \\
\hline $4^{\prime}$ & & 157.7 & $5^{\prime \prime}$ & & 81.5 \\
\hline $5^{\prime}$ & $\begin{array}{l}6.81 \mathrm{~d} \\
(8.4)\end{array}$ & 115.4 & $6^{\prime \prime}$ & & 60.7 \\
\hline $6^{\prime}$ & $\begin{array}{l}7.41 \mathrm{~d} \\
(8.4)\end{array}$ & 130.4 & & & \\
\hline
\end{tabular}

